\title{
Scalable Capacity Estimation for Nonlinear Elastic All-Optical Core Networks
}

\author{
Robert J Vincent, Student Member, OSA, David J Ives, Member, OSA and Seb J Savory, Fellow, IEEE, Fellow, OSA
}

\begin{abstract}
Routing and wavelength assignment (RWA) algorithms must strike a balance between finding routes with high quality of transmission (QoT) and finding routes that will not interfere with allocating future traffic. Too much emphasis on the first will concentrate traffic along major routes causing congestion whilst too much emphasis on the second will cause individual transceivers to operate below their capabilities increasing both cost and power consumption. This paper presents a low-complexity algorithm that shows that focusing on wavelength packing allows for greater overall traffic whilst giving only slight penalties for latency and required transceivers. Our algorithm comfortably outperforms $k$ SP-FF routing for the same complexity and typically betters congestion aware routing whilst reducing complexity. We show these results on 4 simplified networks based on deployed topologies before replicating them on 2,000 artificially generated topologies based on real node locations in Germany and the USA. Capacity for each topology was found with an integer linear program to which our algorithm compares favorably suggesting it provides a scalable alternative to global optimization.
\end{abstract}

Index Terms-Optical fiber communication, networks, routing, capacity.

\section{INTRODUCTION}

$\mathbf{O}$ PTIMAL network design is a delicate balance of economic and physical constraints whilst taking into account future expansion and resilience to inevitable hardware failures [1]. A key aspect is evaluating the maximum traffic a sample network topology can sustain, i.e. its capacity. We quantify capacity as the maximum traffic that can be supported subject to a particular traffic matrix, where the matrix specifies the fraction of total traffic to be routed between each node pair. This is done by assigning a route, wavelength, launch power, modulation format, and any other specifications required of a WDM transceiver. This paper concentrates on the widely studied problem of routing and wavelength assignment. Chlamtac et al [2] showed RWA to be analogous to graph coloring, a well-known NP-hard problem. This means that, assuming $\mathbf{P} \neq \mathbf{N P}$, there exists no polynomial-time algorithm that guarantees an optimal solution. Nevertheless, integer linear programming (ILP) formulations allow such problems to be solved with relative efficiency [3] and have been widely applied to RWA [2], [4]-[6]. ILP formulations do not scale well and are limited to $\sim 20$ node networks. Even at this size, such problems can take an impractically long time to solve

The authors are with the Electrical Engineering Division, University of Cambridge, Cambridge, CB3 0FA, UK. e-mail: rjv36@cam.ac.uk

RJV thanks EPSRC and BT through an iCASE studentship EP/N509103/1 1775341; DJI and SJS thank the EPSRC Programme Grant TRANSNET EP/R035342/1.

Manuscript received 18th Febuary 2019; revised 15th July 2019; accepted 16th September 2019. despite concerted attempts to reduce their difficulty [7]. In the context of network design, we might want to assess a wide variety of topologies and hence an alternative is needed. There are two options that have been widely used for this process: global optimization meta-heuristics and sequential loading. The former solves the full RWA problem using wellknown optimization strategies such as simulated annealing [8], [9], genetic algorithms [10], [11], and various swarmintelligence methods [12]. Unlike the ILP approach, these methods have no guarantee of achieving the optimal solution however they have been shown capable of achieving very good solutions in problems too large for an ILP to be feasible. The latter, sequential loading, simplifies the problem greatly by making lightpath establishment (LE) decisions for one traffic request at a time, rather than all at once. Clearly this has no guarantee of achieving the optimal solution either, however it has the benefit of being equivalent to how service providers might load their networks in practice. This approach removes the combinatorial nature inherent to global optimization of graph problems and their analogues meaning we can scale to arbitrarily large networks with the only significant limitation being the super-linear scaling of shortest path algorithms. Indeed, sequential loading is often simplified further by first making a routing decision and second a wavelength decision [13]. From a list of the $k$ shortest paths, calculated using Yen's algorithm [14], the $k$ SP routing strategy chooses the shortest path with a viable wavelength option. This is combined with a wavelength assignment strategy such as first fit (FF) which assigns the first viable wavelength along a given route. Together, $k \mathrm{SP}-\mathrm{FF}$ provides a convenient and simple baseline that performs well in most cases. This can be improved upon by using congestion aware routing [15] which weights routes on a combination of their length and their congestion. More complex approaches, such as max sum and relative capacity loss [16], [17], might perform even better in some cases but they require knowledge of upcoming traffic requests as well as pre-determined route assignments, and are therefore not able to assign a single request in isolation. This limitation means we shall not consider them further.

This paper reverses the standard approach to sequential loading by focusing first on assigning demands into as few wavelengths as possible and second on finding an appropriate route. We shall see that this outperforms $k \mathrm{SP}-\mathrm{FF}$ with no increase in complexity and trades blows with congestion aware routing whilst reducing complexity. We also compare performance to the optimal ILP solution and show that our algorithm provides a scalable alternative to estimate network capacity for network design applications. We confirm this 
TABLE I

SiMULATION PARAMETERS

\begin{tabular}{l|c|l}
\hline \hline Attenuation $\left(\alpha_{\mathrm{dB}}\right)$ & 0.22 & $\mathrm{~dB} \cdot \mathrm{km}^{-1}$ \\
Nonlinear coefficient $(\gamma)$ & 1.3 & $\mathrm{~W}^{-1} \mathrm{~km}^{-1}$ \\
Chromatic Dispersion $(D)$ & 16.7 & $\mathrm{ps} \cdot \mathrm{nm}^{-1} \cdot \mathrm{km}^{-1}$ \\
Span Length $\left(L_{\text {span }}\right)$ & 80 & $\mathrm{~km}$ \\
\hline Num Wavelengths $(\mathrm{W})$ & 80 & - \\
Channel Launch Power & -1 & $\mathrm{dBm}$ \\
Symbol Rate $(R)$ & 32 & $\mathrm{GBd}$ \\
Effective Symbol Rate, post-FEC & 25 & $\mathrm{GBd}$ \\
\hline EDFA Noise Factor & 5 & $\mathrm{~dB}$ \\
\hline \hline
\end{tabular}

result across 2,000 topologies first introduced in [18]. We shall begin by outlining the simulation parameters and the network topologies used in Section II. We then describe previous approaches in more detail and go on to discuss our contributions in Section III. We show the operation of our algorithm and compare its performance to previous attempts in Section IV. We discuss our results and suggest further work in Section V and summarize our findings in Section VI.

\section{Methodology}

In this section we first describe the simulation parameters and proceed to estimate transmission signal-to-noise ratio (SNR) using the Gaussian Noise (GN) model [19], [20] for the central WDM channel in a fully loaded span and extrapolate this for lightpaths of arbitrary distance. After this we describe the network topologies used and show how we created 2,000 topologies based on the node locations of real networks.

The simulation parameters used in this experiment are shown in Table I. We assume a uniform transmitter power of $-1 \mathrm{dBm}$ per channel which was found to be near-optimal with our assumptions. The other values are typical of modern transmissions over standard single mode fiber (SSMF) although we use a uniform span length to simplify SNR estimates. We assume a $32 \mathrm{GBd}$ transmission with a $20 \%$ hard-decision FEC which, along with other overheads, results in an effective symbol rate of $25 \mathrm{GBd}$. We assume attenuation is due to the fiber alone but a value of $0.22 \mathrm{~dB}$ implicitly includes splice defects. We assume ROADMs to be lossless, as this reduces the dependence of optimum launch power on path length, however as each routing algorithm uses these same assumptions it has no effect on their relative effectiveness.

\section{A. Estimating Transmission SNR}

We first estimate SNR for the worst-case central channel, of 80 , in a fully loaded span and then extrapolate this to $n$ spans. We assume amplifiers are placed at the end of each span and are configured such that they precisely compensate the attenuation in the preceding fiber, meaning amplified spontaneous emission (ASE) noise is the same for all spans in a lightpath. Nonlinear noise is calculated using the GN-model [19] with contributions from self- and cross-phase modulation (SPM \& XPM) in WDM channel $i$ in a single span given by:

$$
N_{i}=p_{i} \sum_{j \in\{1 . . W\}} X_{i j} p_{j}^{2}
$$

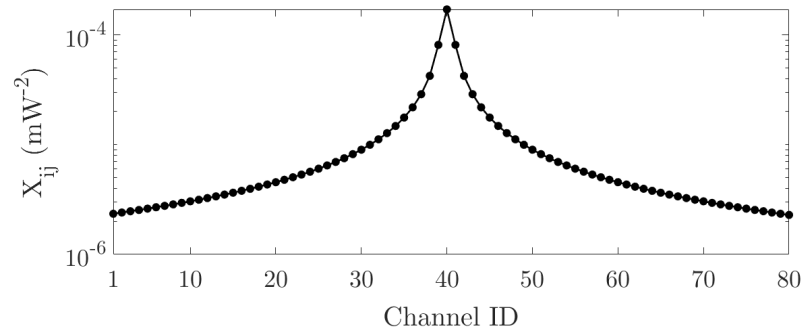

Fig. 1. Nonlinear interference strength between the central, 40th channel and all other channels (including itself) in a fully loaded WDM span.

where $p_{i}$ is the transmitted power in the $i$ 'th WDM channel and $X_{i j}$ denotes the strength of interaction between channels with $i=j$ being from SPM and $i \neq j$ from XPM. Four-wave mixing is neglected because of its small contribution in the context of large $18 \mathrm{GHz}$ guardbands [21]. We can also neglect inter-channel stimulated Raman scattering because we are restricted to $4 \mathrm{THz}$ total spectrum and $\sim 18 \mathrm{dBm}$ total power. This indicates a change in SNR of approximately $0.2 \mathrm{~dB}$ which we deem suitability covered by the worst-case channel assumption [22]. As we assume each channel has the same bandwidth $X_{i j}$ depends only on the separation of frequencies, i.e. $X_{i j}\left(\nu_{i}, \nu_{j}\right)=X_{i j}\left(\left|\nu_{i}-\nu_{j}\right|\right)$. When considering the central channel, this allowed us to pre-calculate 41 values denoting the interaction to itself and its neighbours. Given we are using an even number of channels, there is no definitive 'central' channel, so we choose channel 40 . Hence, the worst-case nonlinear interference estimate for every channel $i$ over a single span is assumed to be:

$$
N_{i}=p_{40} \sum_{j \in\{1 . . W\}} X_{40, j} p_{j}^{2}
$$

with the coefficients $X_{40, j}$ shown in Fig. 1 .

We can extrapolate this for $n$ spans by introducing the nonlinear efficiency factor $\varepsilon$, which defines the extent to which SPM is coherent [20], whilst assuming this value is 0 for the XPM term:

$$
\begin{aligned}
N_{i}(n)= & n^{1+\varepsilon} X_{40,40}\left(p_{40}\right)^{3}+n p_{40} \sum_{j \in\{1 . . W \backslash 40\}} X_{40, j} p_{j}^{2} \\
& =n p^{3}\left(n^{\varepsilon} X_{40,40}+\sum_{j \in\{1 . . W \backslash 40\}} X_{40, j}\right)
\end{aligned}
$$

where $\varepsilon$ was calculated previously to be 0.2186 [23], and Eq. (4) follows from the requirement that all channels carry the same power, namely $p$.

Linear noise is assumed to be ASE noise from the aforementioned amplifiers counteracting losses from fiber attenuation only. We model ASE noise from a single EDFA from the standard formula, and given there is one at the end of each span we can extend it for $n$ spans by a simple multiple:

$$
N_{\mathrm{ASE}}(n)=n 10^{\mathrm{NF} / 10} h \nu_{c} R \cdot 10^{L_{\mathrm{span}} \alpha_{\mathrm{dB}} / 10}
$$

where $\nu_{c}$ is the central frequency $(193.5 \mathrm{THz}), \mathrm{NF}$ is the noise factor of all amplifiers, $R$ is the symbol rate of the transmission, and the final term is the gain of each amplifier which is equivalent to the loss in a span of fiber. 


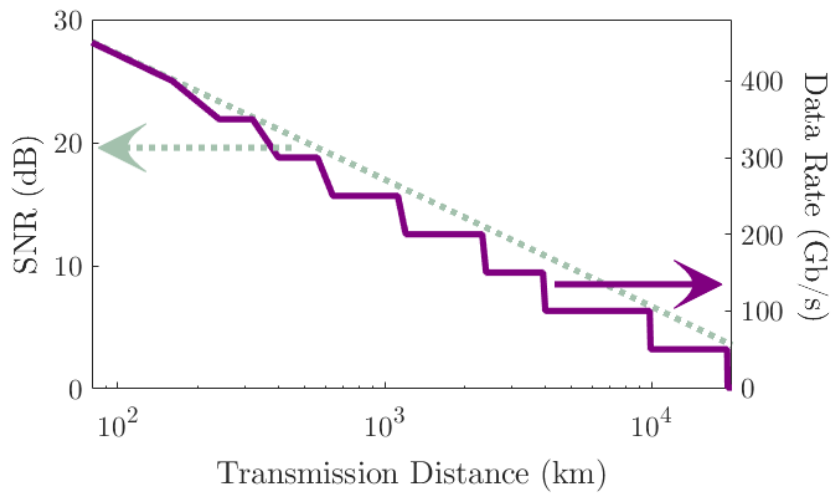

Fig. 2. Pre-calculated SNR (dashed, left axis) and maximum transmission data rates (solid, right axis). Maximum reach for PM-BPSK was 19,200 km, or $24080 \mathrm{~km}$ spans.

We estimate the required SNR of each $m$-QAM format by restricting errors to the nearest neighbours of the intended symbols; this approach, [6] adapted from [24], is exact for BPSK and QPSK but introduces small errors in higher modulation formats because a symbol error can result in a variable number of bit errors depending on where in the constellation the error happens. With 80 identical channels, we found the optimum Tx power to be $-1.28 \mathrm{dBm}$. This is very similar to the $-1 \mathrm{dBm}$ Tx power we used initially and power optimization has been shown [25], [26] to have a relatively small effect and we shall therefore keep this uniform value for simplicity.

We then assume a hard-decision continuously interleaved BCH FEC with $20 \%$ overhead that gives a net coding gain of $10.5 \mathrm{~dB}$ [27], [28]; this allows a transmission with a BER of $1.5 \%$ to be corrected to 'error free'. The calculated SNRs for transmission across many spans are shown in Fig. 2, as well as the maximum possible data rate. It should be noted that we prefer increased SNR margin to unnecessary data transmissions and hence lightpaths may fall below the data rates shown when a lower order modulation format can meet the requested demand. The high spectral efficiency combined with long reach is not feasible with current commercial equipment. We assume near-perfect transceivers under the assumption that technology will continue to advance and in the knowledge that our overly optimistic SNR estimates with have little effect on the relative performance of the routing algorithms presented.

\section{B. Network Topologies}

To compare algorithm performance we require a range of network topologies to ascertain whether differences are due to the RWA algorithms or simply a quirk of a particular network. Clearly if a service provider wants to optimize their own network this is not necessary but the scope of this work is to find a high-performing algorithm on an arbitrary topology. We use standard topologies, common throughout the literature, on which to compare algorithms in addition to creating our own topologies based on these examples.

We begin by introducing four networks based on real-world topologies. Perhaps the most popular of these is NSFnet used with either 21 links [4], [6] or with 22 [15]; the extra link

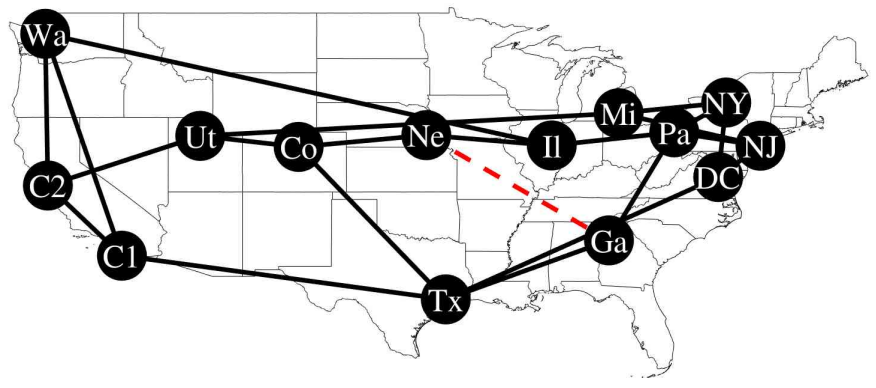

Fig. 3. Topology of NSFnet within the USA with 21 and 22 links. The extra link, connecting $\mathrm{Ne}$ to $\mathrm{Ga}$, is highlighted. When referring to NSFnet, we typically mean the 22 link version, but use the names NSFnet21 and NSFnet22 when deemed necessary.

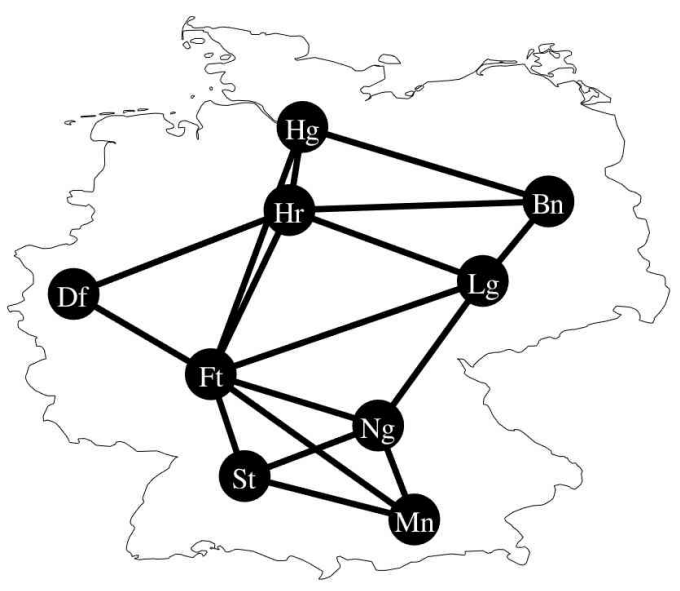

Fig. 4. Topology of DT9 within Germany [29].

makes the network more homogeneous by having only 3 and 4 degree nodes. By default we use NSFnet to refer to the 22 link version but when both versions are used we shall use NSFnet 21 and NSFnet22 to avoid ambiguity. We shall use the 22 link version, i.e. with the extra link, shown in Fig. 3 to represent a continental-scale network.

We move on to national-scale networks from (relatively) small European countries. These topologies, based on those of British Telecom plc (BT) in the UK and Deutsche Telekom AG (DT) in Germany, are more dense than NSFnet understandably given the population densities of the countries they connect. We have 2 networks from DTAG one with 9 nodes [29] and another with 14 [6]; these are shown in Figs. 4 and 5, respectively.

Link lengths are calculated from the great circle distance between relevant nodes (using the haversine formula and their longitude and latitudes). These distances, $D_{\text {hav }}$, are then adjusted to fiber distance to give a more realistic estimate of the length of fiber required if the link were to be built [30]:

$$
D_{\text {fiber }}= \begin{cases}1.5 \cdot D_{\text {hav }} & \text { if } D_{\text {hav }}<1000 \mathrm{~km}, \\ 1500 \mathrm{~km} & \text { if } 1000 \mathrm{~km} \leq D_{\text {hav }} \leq 1200 \mathrm{~km}, \\ 1.25 \cdot D_{\text {hav }} & \text { if } D_{\text {hav }}>1200 \mathrm{~km},\end{cases}
$$

BT does not publish the exact locations of the nodes on their test topology therefore we take the link lengths provided by [31] rather than employing the haversine approach. There are 


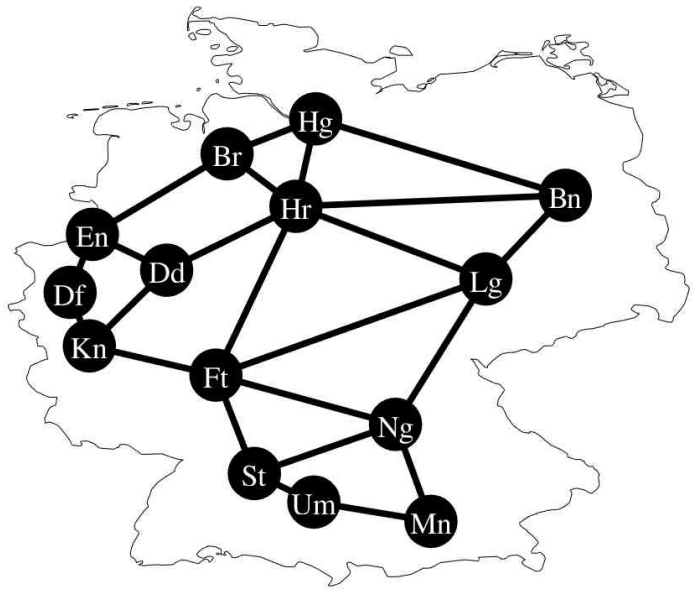

Fig. 5. Topology of DT14 within Germany [6].

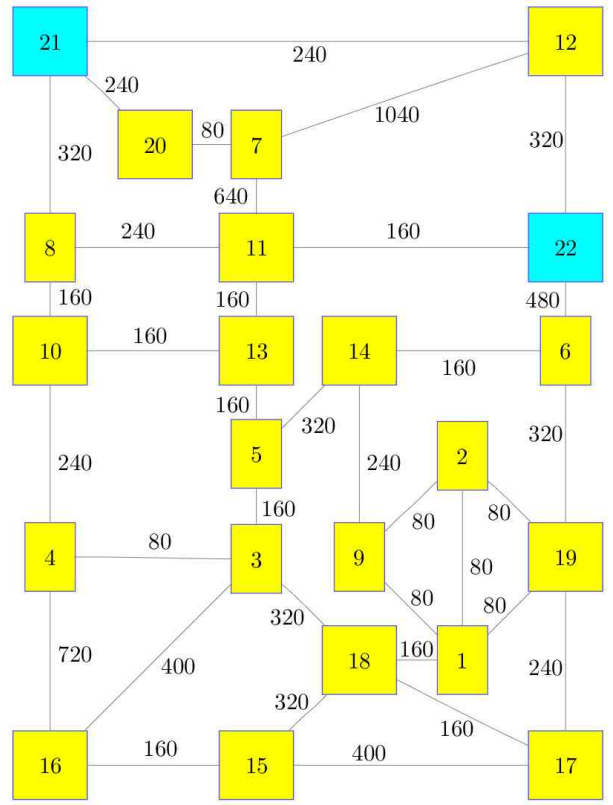

Fig. 6. Topology of BT22. All link lengths are in $\mathrm{km}$ and node positions are not representative of their geographic locations. Nodes 21 and 22 (in blue) do not produce or consume traffic and serve only to aid connections between other nodes.

two additional quirks: first is that certain nodes (presumably in central London) are very close together, e.g. $2 \mathrm{~km}$, which is problematic given our uniform span length is $80 \mathrm{~km}$. We solve this by inelegantly but pragmatically assuming that the minimum length of any link is a single span. The second is that 2 nodes of the 22 do not produce traffic and are simply there to help route traffic: a relic from when reach was a significant problem even in small countries. We show a version of the BT22 topology in Fig. 6.

We shall now describe more networks, first discussed in [18], that were generated to give a comparison of algorithms on as wide a range of topologies as possible. To this end we take the nodes of NSFnet, Fig. 3, and DT9, Fig. 4, and connect nodes by selecting links at random, a process popular with generating random graphs [32], [33]. Because we are interested not in random graphs but topologies for communication networks we add further restrictions. The most obvious of these is that the network must be connected - i.e. there exists a path connecting every node to every other node on the network - so we shall discuss the challenge of making the topology 'sensible'. We are considering core networks so there might be some inclination towards homogeneity in that all nodes and links are of roughly equal importance and that the network is resistant to fiber cuts. We might also require an efficient use of fiber so that each link is serving a purpose either to connect nodes or to shorten the distance between them. These requirements are deliberately vague to highlight that these choices are somewhat arbitrary and that we are not here concerned with generating perfect networks, rather we want a reasonable set of networks on which to compare algorithms.

To generate reasonable network topologies we created a genetic algorithm (GA) with a cost function given in Eq. (7), where $\mathcal{G}$ is the graph; $\mathcal{G}_{i j}$ is the link length between nodes $i$ and $j$ if such a link exists, and zero otherwise; and $\mathcal{D}_{i j}(\mathcal{G})$ finds the shortest path distance between nodes $i$ and $j$. This cost function expresses the product of graph diameter, the longest shortest path, and the total fiber length.

$$
f(\mathcal{G})=\max _{i<j}\left\{\mathcal{D}_{i j}(\mathcal{G})\right\} * \sum_{i<j} \mathcal{G}_{i j}
$$

The GA then finds networks that minimise Eq. (7) subject to the following constraints:

- the graph is connected

- the degree of all nodes is between 2 and 5, inclusive

The first of these needs no explanation and the second ensures that no nodes can act as a 'central hub' by restricting the maximum node degree and that a single fiber cut can not isolate a node from the rest of the network. We then ran the GA and used its archive to populate a set of 1,000 unique networks based on NSFnet and the same number based on DT9, these networks sets are refered to as NSFTEST and DT9TEST, respectively. Some example topologies are given in the Appendix.

\section{OfFline Lightpath Assignment Algorithms}

This section first describes previous work in this area and goes on to describe how to adapt uniform sequential traffic to give more consistent traffic matrices. We then introduce the wavelength and routing assignment algorithm, $\mathrm{FF}-k \mathrm{SP}$, which we developed to better utilize network resources by focusing on wavelength packing over spectral efficiency.

\section{A. Past Developments}

A sequential loading template is given in Algorithm 1 and all algorithms discussed below follow this basic outline. First, a demand is requested and the network state at that moment is inspected. If a lightpath connecting the relevant nodes is already in place, it can try to upgrade the modulation format subject to SNR overhead; if possible, we upgrade the format and sample another demand. If not, we search for a lightpath that is capable of the required data rate and that is not blocked 


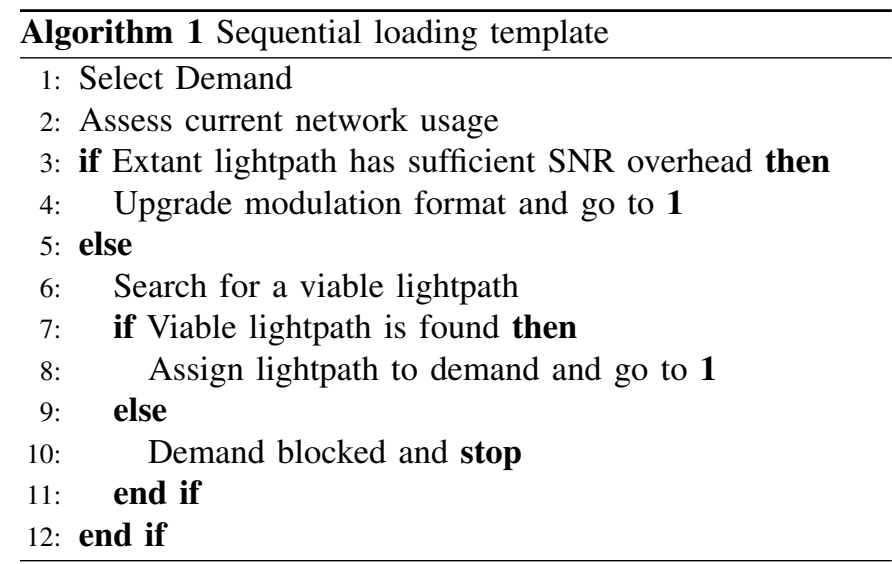

by other demands on the network; this search strategy is where the algorithms differ. If a lightpath is found, we assign it to the current demand and again sample another. If no such lightpath is found the demand is blocked and we stop the simulation. We stop the simulation at first blocking because once a lightpath is established we do not expect it to be turned off and, given we search all possible route/wavelength combinations for a demand, if no viable lightpath is found now, no such lightpath will be found in the future.

We assume that each network link consists of two fibers. This allows lightpaths assigned for $\mathrm{A} \rightarrow \mathrm{B}$ to use one set of fibers and lightpaths for $\mathrm{B} \rightarrow \mathrm{A}$ to use the same wavelength assignments but on another, independent set of fibers. This allows bi-directional traffic to be assigned whilst maintaining wavelength continuity.

Shortest Path - First Fit (SP-FF) is the simplest algorithm we consider and operates by only allowing demands to use the shortest path. SP-FF searches wavelength channels along the shortest path and assigns the first viable lightpath it finds. Clearly this is less than ideal, not only for minimizing congestion, but for resilience. With only one route option per demand, in the event of a single fiber cut at least 1 node pair will be completely disconnected. For this reason, we can extend SP-FF to $k \mathrm{SP}-\mathrm{FF}$ [1] which, if the shortest path is blocked, moves on to the second shortest path. This continues until either a viable lightpath is found or the search exhausts all $k$ path options and the demand is blocked.

Wavelength assignment strategies that perform significantly better than first fit typically require pre-assigned route choices for all the request demands (e.g. Max Sum [5], [16] and Relative Capacity Loss [17]). Without knowing route choices in advance we are left with three major alternatives: random wavelength assignment (Rand), most used (MU), and least used (LU). Rand searches for viable wavelengths in a random, non-repeating sequence; MU searches the wavelength used on the most links first before moving on to less used wavelengths; and LU searches the least used wavelength on the network before it searches more popular wavelengths. The performance hierarchy of these approaches is well understood with MU performing best but only slightly better than FF. Rand is significantly worse with LU similarly poor [1].

An alternative routing assignment strategy can be found by considering instantaneous network utilization. Congestion aware routing (CA) extends $k \mathrm{SP}$ routing by searching routes in an order determined not only by path length but network congestion [15]. We quantify congestion on a particular link as the proportion of unused wavelengths on that link and for a particular path as the proportion of unused wavelengths on the set of links traversed by that path. Having calculated path congestion, we can find the ratio of this with path length and assign this value to the path to indicate its suitability. We then sort our $k$ route options subject to this weighting, instead of simply path length, and search them accordingly. This was shown to perform noticeably better than $k \mathrm{SP}$ routing [15]. We can improve the weighting slightly by including the path data rate because a shorter path on which a better modulation format is available may well be preferable to a slightly less congested path with worst spectral efficiency. This leads to the link weighting given in Eq. (8).

$$
\mathrm{LW}_{\mathrm{CA}}=\frac{\text { Fraction occupied wavelengths }}{\text { Link Length }(\mathrm{km})}
$$

To calculate the path weighting we simply sum the relevant link weights and multiply by the path data rate.

By combining both routing assignment and wavelength assignment strategies we have the congestion aware most used algorithm (CA-MU). This is the best RWA approach that maintains flexibility by not requiring pre-assigned routes or knowledge of upcoming traffic requests.

To find an optimal solution we can not use the sequential approaches and are forced to resort to a global optimization approach solved with either an ILP [6] or other heuristics [11], [12]. We shall use the ILP-based method from [6] to find the capacity of a network under uniform traffic. This uses IBMs CPLEX API to solve a binary ILP which optimizes the vector $\delta$ where $\delta_{d k w}$ is 1 if demand $d$ is assigned a lightpath using route option $k$ and wavelength $w$; and 0 otherwise. The optimization first uses relaxed wavelength continuity constraints to find an upper bound and then attempts to reach this upper bound with full wavelength continuity in place. It is this stage that typically requires the bulk of the computation time.

In effect, this stage does not maximize uniform traffic rather it maximizes the minimum traffic between node pairs. These are almost equivalent however using the latter approach means that although all demands are above some traffic threshold, some demands can have more traffic than others. This uses more transceivers than necessary to achieve the same uniform traffic level. For this reason, a further stage was introduced to minimize the number of transceivers whilst fixing the minimum traffic level between node pairs. This stage is very fast hence increasing the complexity of cost function is relatively cheap computationally. The final cost function used was:

$$
f(\boldsymbol{\delta})=\sum_{d k w} \delta_{d k w} \cdot(A+B+C)
$$

where

$$
A=1000 ; B=\frac{D_{d k}}{100}\left(\frac{\mathrm{SNR}_{\text {thres }}}{\mathrm{SNR}_{d k}}\right)^{2} ; C=\frac{w}{1000} B
$$




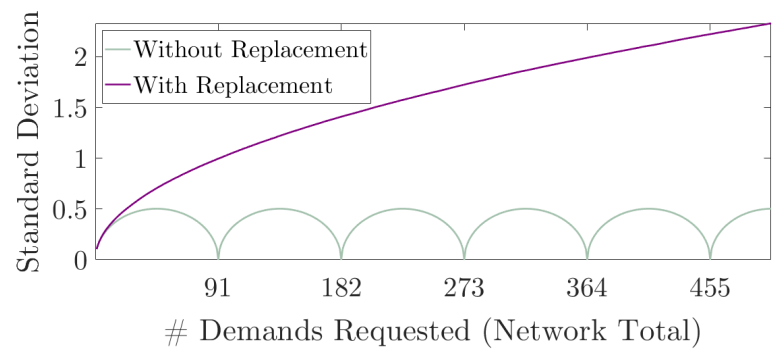

Fig. 7. Standard deviation of the number of demands requested between each node pair plotted against the number of demands requested on the network as a whole. We show the effect of selecting demands uniformly at random with and without replacement on a 14 node network with 91 demands. Selection with replacement results in increasing variance between demands however selection without replacement results in periodic behavior that returns to zero every 91 traffic units because a this point all demands have the same traffic level.

with $D_{d k}$ and $\mathrm{SNR}_{d k}$ the distance and SNR of demand $d$ using route $k$, respectively. $\mathrm{SNR}_{\text {thres }}$ is the minimum required SNR for the modulation format used by the lightpath $\delta_{d k w}$. As $A>>B>>C$ by minimizing this cost function we perform the following: $A$ minimizes the number of active transceivers; $B$ penalizes the use of long routes with small SNR margins that will require higher transmit power and cause NLI on other channels; and $C$ seeks to cluster these high transmit power lightpaths into the same few wavelengths which can then be maximally separated reducing the risk of two high power lightpaths sharing adjacent frequency slots. The values chosen are such we can never turn on more transceivers to reduce the number of high transmit power lightpaths.

\section{B. Uniform Traffic}

Most RWA simulations assumed uniform traffic between nodes however only global optimization methods are able to guarantee this as the final traffic ratio. Sequential approaches typically select demands with uniform probabilities however blocking occurs long before the variance in the traffic for each demand shrinks. This results in some ambiguity: is the resulting traffic level a consequence of the lightpath assignment decisions or randomness in the traffic matrix? To answer this, and provide a better comparison to ILP results, we suggest using uniform selection without replacement: it is only after all demands have been selected once that we allow them to be selected for a second time. This means that the maximum difference between traffic levels is 1 demand. This can be seen in Fig. 7 where the standard deviation of traffic for demands on NSFnet is plotted against the number of traffic requests. For a 14 node network, with 91 demands, variance between traffic levels increases with total traffic for uniform selection with replacement (solid line): the more traffic is on the network, the less uniform it becomes. Whereas selecting without replacement (dashed line) results in oscillatory behavior where the variance reaches a maximum when half the demands have traffic $T$ and the other $T+1$, and declines until every demand has the same traffic, in this case $T+1$; this repeats every $N(N-1) / 2$ demands, which in this example is multiples of 91.

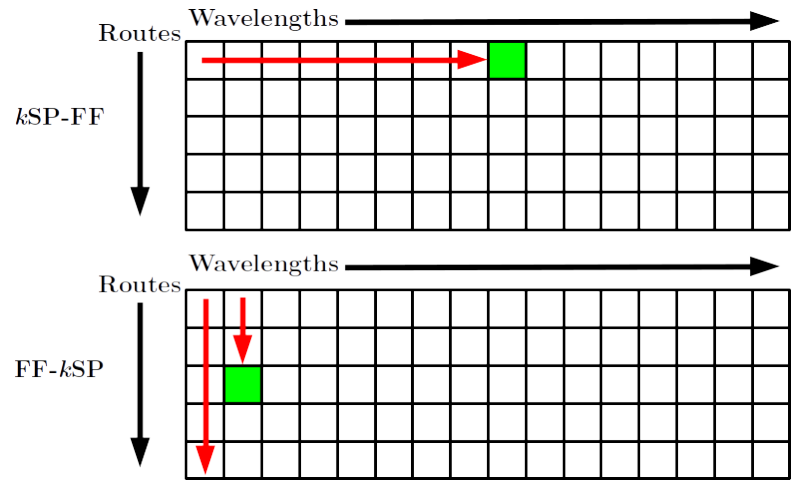

Fig. 8. Operational comparison of the well-known $k$ SP-FF to our algorithm $\mathrm{FF}-k \mathrm{SP}$. $k \mathrm{SP}-\mathrm{FF}$ searches all possible wavelengths to assign the shortest possible route, whereas $\mathrm{FF}-k \mathrm{SP}$ searches all possible routes to assign the first possible wavelength.

This approach gives a more uniform final traffic matrix which not only allows better approximation of the desired traffic matrix but enables a direct comparison to ILP solutions.

\section{Wavelength and Routing Assignment}

If a wavelength remains unused on all links any path on the network can be routed along that wavelength without contention. Packing traffic into as few wavelengths as possible is beneficial because it follows that more wavelengths remain vacant. We must balance this with the spectral efficiency of each lightpath, i.e. fewer efficient lightpaths will carry the same traffic as more inefficient lightpaths. Typical routing approaches [1], [15] implicitly assume that spectral efficiency is more important by opting for a longer route only in cases of congestion on the shortest path. We suggest preemptively using longer routes to increase packing efficiency which, as we shall show, allows more spectrum to be supported (albeit this operating less efficiently).

We show the operation of our algorithm FF- $k$ SP in Fig. 8, in comparison to the aforementioned $k \mathrm{SP}-\mathrm{FF}$. In both cases the $k$ route options are sorted in length order and wavelengths in some fixed order. $k$ SP-FF attempts to assign a lightpath along the shortest possible path, whereas FF- $k \mathrm{SP}$ attempts to assign the lightpath on the first possible wavelength. The arrows indicate the different search direction and both algorithms assign the first viable route/wavelength pair they find.

\section{RESUlTS}

We shall begin by highlighting the operation of $\mathrm{FF}-k \mathrm{SP}$ in comparison to $k \mathrm{SP}-\mathrm{FF}$ on NSFnet. After this, we shall compare the traffic before blocking with a variety of algorithms on the networks described above. We go on to compare the sequential solution to that obtained by an ILP for cases that achieved capacity.

\section{A. $F F-k S P$ in Action}

Before discussing increased traffic levels, it is useful to 'see' our algorithm working in practice. In Fig. 9 we compare the network utilization of $k \mathrm{SP}-\mathrm{FF}$ and $\mathrm{FF}-k \mathrm{SP}$ after routing 


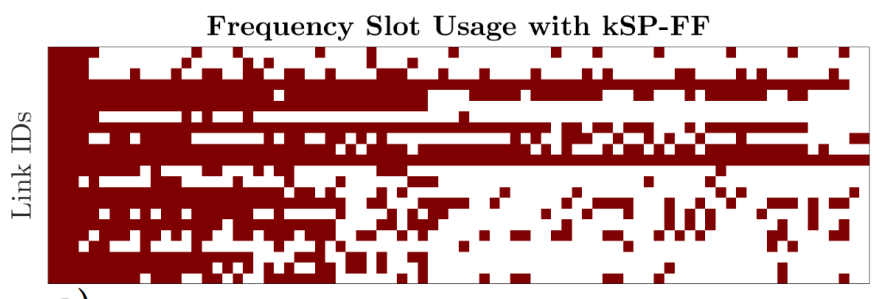

a)

Wavelength Channels

Frequency Slot Usage with FF-kSP

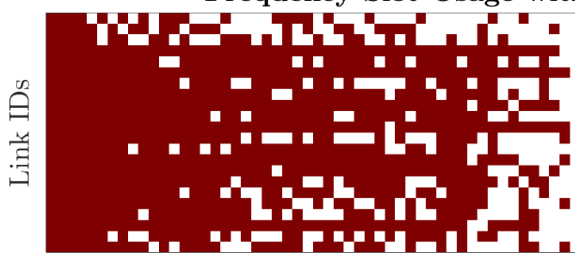

b)

Wavelength Channels

Fig. 9. Occupancy of frequency slots for $k \mathrm{SP}-\mathrm{FF}$ (a) and FF- $k \mathrm{SP}$ (b) on NSFnet for the same traffic level, namely $81.9 \mathrm{~Tb} / \mathrm{s}$. $k$ SP-FF uses fewer frequency slots, 801 to 827 , indicating higher spectral efficiency but spreads traffic less evenly across network links than FF- $k$ SP.

the same amount of traffic on NSFnet with $k$ set to 5 i.e. each demand has up to 5 route options available. We plot the frequency slots on the network highlighting those that are assigned lightpaths and leaving unassigned slots blank. We see that $k$ SP-FF, Fig. 9a, heavily utilizes certain links over others because shortest paths tend to use the topological centre of a network as it is often the most direct route. By selecting the shortest path in all cases unless it is blocked, these central links become quickly congested to enable higher spectral efficiency in the short term. The links that are most heavily used connect $\mathrm{C} 2 \rightarrow \mathrm{Ut}$ and $\mathrm{Co} \rightarrow \mathrm{Ne} \rightarrow \mathrm{Il} \rightarrow \mathrm{Pa}$, which are indeed the most popular links selected amongst all possible shortest paths. By comparison FF $-k \mathrm{SP}$, in Fig. 9b, spreads usage more evenly across all network links. There remains a hierarchy with some links more popular than others but the difference is far less stark than with $k \mathrm{SP}-\mathrm{FF}$. This is achieved by using slightly longer routes and, as expected, requires the use of more spectrum overall but the difference is just over $3 \%$ in this example while the difference in congestion is much more significant. We see that using $k \mathrm{SP}-\mathrm{FF}$ certain links are already blocked while only 50 of 80 wavelengths carry the same traffic with $\mathrm{FF}-k \mathrm{SP}$.

We choose a value of $k$ above which the algorithm appeared to be stable on the 4 topologies in Figs. 3 to 6 . We plot this relationship in Fig. 10 where we see the algorithm plateau for values above about 5 . We shall investigate later in the paper whether this trend is valid across a wider range of topologies.

Inherent to its operation, $\mathrm{FF}-k \mathrm{SP}$ uses longer routes than $k \mathrm{SP}-\mathrm{FF}$ and this could result in additional latency. We show this effect in Fig. 11a where we plot the tail distribution of route distances on NSFnet across 10,000 iterations. The longest route used by $k \mathrm{SP}-\mathrm{FF}$ was $7520 \mathrm{~km}$ across all runs whereas $\mathrm{FF}-k \mathrm{SP}$ assigned routes exceeding this length in over $1 \%$ of cases. Figure $11 \mathrm{~b}$ shows the worst case latency for every node pair compared to that of the shortest path. FF- $k$ SP can result in lightpaths that are much longer than the shortest

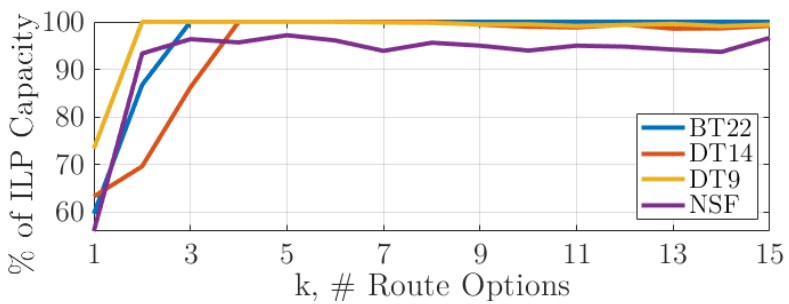

Fig. 10. Maximum traffic levels achieved with $\mathrm{FF}-k \mathrm{SP}$ with various values of $k$ relative to the optimal capacity calculated with an ILP for the 4 networks in Figs. 3 to 6 . This appears to justify the initial choice of $k=5$ as the algorithm plateaus above this value.

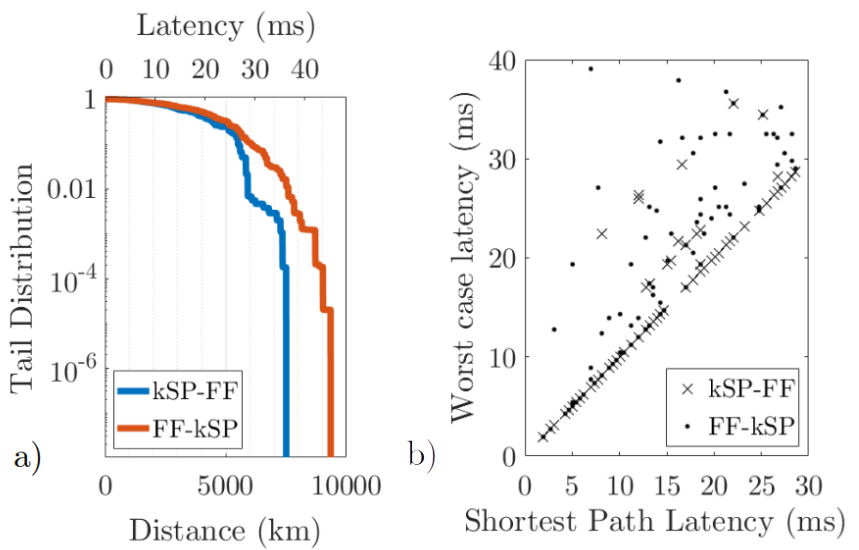

Fig. 11. a) The probability a route choice exceeds a certain distance using $k \mathrm{SP}-\mathrm{FF}$ and $\mathrm{FF}-k \mathrm{SP}$ for 10,000 iterations on NSFnet. b) The worst case latency for every node pair on NSFnet compared to the shortest path. Latency is calculated assuming a fibre refractive index of 1.45 with no additional delays.

path with a latency penalty of $\sim 30 \mathrm{~ms}$; this corresponds to around 2 frames on a standard $60 \mathrm{~Hz}$ monitor in additional to all other delays. For services that require the end user to interact, perhaps cloud-computation for video games or even aspects of the tactile Internet initiative, any additional latency must be avoided and even this small increase may tilt a service from viable to unusable. Fortunately, much IP traffic is less sensitive to latency: high fidelity video streams, for instance, require high bitrates but an additional pause before playing is borderline unnoticeable. Therefore the latency increase on some lightpaths can be mitigated by ensuring latency-sensitive requests use the shortest possible path whereas less sensitive traffic can take the longer route options.

\section{B. Effect on Traffic Levels}

We shall now compare FF- $k$ SP to other sequential algorithms as well as to a global optimization approach with an ILP. In Fig. 12 we compare performance of three sequential algorithms and the optimal capacity with uniform traffic achieved with an ILP on the four real-world network topologies shown in Figs. 3 to 6. For the sequential algorithms we plot mean network traffic and for the ILP we show the optimal traffic achieved with uniform traffic. The ILP result shows the target value and we can only achieve higher traffic by deviating from a uniform traffic matrix. With our traffic 


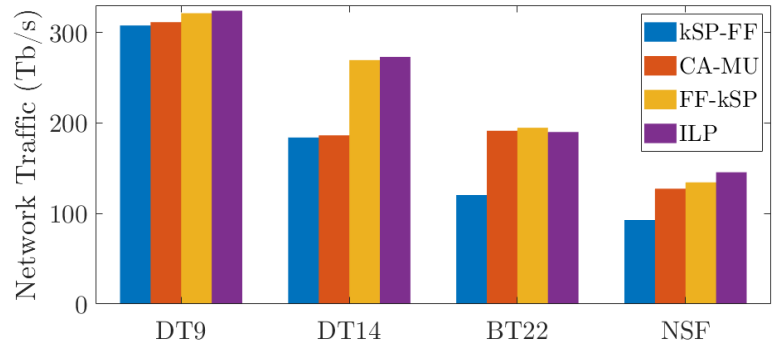

Fig. 12. Mean traffic on 4 real-world topologies in Figs. 3 to 6 with 5 routes options along with the ILP optimal capacity with uniform traffic between nodes.

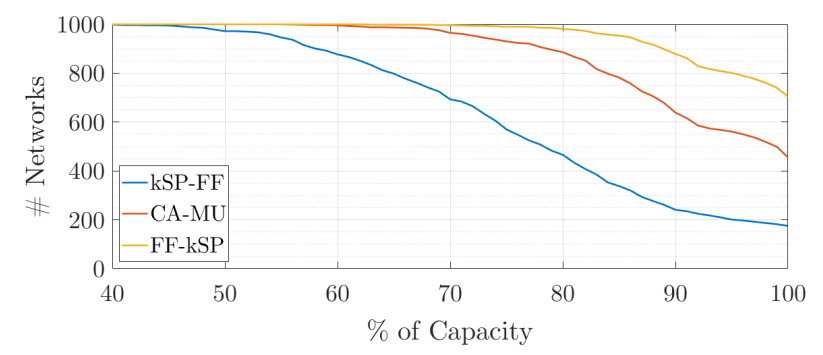

Fig. 13. Showing the number of networks from NSFTEST that exceed some percentage of optimal ILP capacity for 3 sequential algorithms. We see that $\mathrm{FF}-k \mathrm{SP}$ comfortably outperforms its competition, with CA-MU showing a distinct improvement over $k \mathrm{SP}-\mathrm{FF}$.

sequence, the upper bound for sequential traffic is therefore above the ILP limit: it ranges from $1 \%$ higher for DT9 to $10 \%$ higher for $\mathrm{BT} 22$. We see that $\mathrm{FF}-k \mathrm{SP}$ achieves higher traffic than either $k \mathrm{SP}-\mathrm{FF}$ or $\mathrm{CA}-\mathrm{MU}$. The effect is minor for DT9, which is very close to optimal capacity with $k \mathrm{SP}$ FF anyway, and more significant for the larger networks. DT14 sees little improvement from switching from $k$ SP-FF to congestion aware routing but with $\mathrm{FF}-k \mathrm{SP}$ performance is near-optimal. On BT22, both CA-MU and FF- $k$ SP achieve optimal capacity consistently. On NSFnet, we see a small improvement from $k \mathrm{SP}-\mathrm{FF}$ to $\mathrm{CA}-\mathrm{MU}$ and again to $\mathrm{FF}-$ $k \mathrm{SP}$ even this does not achieve optimal ILP capacity. This means that $\mathrm{FF}-k \mathrm{SP}$ outperforms $k \mathrm{SP}-\mathrm{FF}$ and $\mathrm{CA}-\mathrm{MU}$ on all four networks. The performance difference over CA-MU varies significantly from network to network but there are no networks on which CA-MU outperforms FF- $k$ SP.

We now compare performance of the algorithms on DT9TEST and NSFTEST. Clearly, we are not expecting to achieve the ILP capacity in all cases because of the limitations inherent to sequential loading but we are interested in the likelihood of a 'good' estimate of capacity which we have previously defined as better than $90 \%$ of capacity. We plot the probability that a given algorithm achieves more than a certain percentage of the optimal ILP capacity. This probability is across a set of 1,000 network topologies and takes the best solution from 1,000 pseudo-random traffic sequences for each network. We see the results for NSFTEST in Fig. 13 and this shows that $\mathrm{FF}-k \mathrm{SP}$ is more likely to be closer to capacity than $\mathrm{CA}-\mathrm{MU}$ and that both are significantly better than $k \mathrm{SP}-$ FF. Worst-case performance is especially poor for $k \mathrm{SP}-\mathrm{FF}$

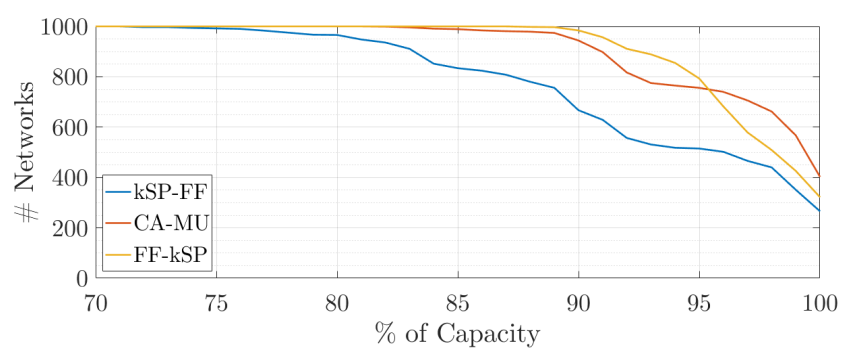

Fig. 14. Showing the number of networks from DT9TEST that exceed some percentage of optimal ILP capacity for 3 sequential algorithms. FF$k \mathrm{SP}$ outperforms $\mathrm{CA}-\mathrm{MU}$ in the worst-case (a $12 \%$ deficit compared to $18 \%$ ), but the latter algorithm is more likely to achieve traffic levels very close to capacity, i.e. greater than $95 \%$ of optimal. As expected, $k \mathrm{SP}-\mathrm{FF}$ performs worse than either alternatives tested.

with one network not even achieving $40 \%$ of capacity. This improved to $55 \%$ with CA-MU and $62 \%$ with FF- $k$ SP.

In Fig. 14 we show the same results for DT9TEST; the results here are more nuanced. Again, FF- $k \mathrm{SP}$ and $\mathrm{CA}-$ MU easily outperform $k$ SP-FF however on these networks CA-MU is more likely to achieve a very high fraction of capacity. This effect only becomes apparent when we are already achieving over $95 \%$ of capacity. FF- $k$ SP performs better in the worst-case with a minimum shortfall of $12 \%$ across the 1,000 networks compared to $18 \%$ for CA-MU and $28 \%$ with $k$ SP-FF.

We also note that the smaller networks of DT9TEST are more likely than NSFTEST to achieve a 'good' estimate of capacity $-99.7 \%$ to $87.9 \%$ with FF- $k$ SP achieve $>90 \%$ of capacity - but that the larger networks are more likely to actually achieve capacity $-70.3 \%$ to $42.6 \%$.

Using the 2,000 networks, we can ask if the relationship shown in Fig. 10 is valid for an arbitrary topology. We see the results in Fig. 15 with the upper section showing NSFTEST and the lower DT9TEST. We plot the probability that a network from the set of 1,000 exceeds 80, 90, and $95 \%$ of ILP capacity for a given $k$. On DT9TEST we see a very similar result to that which we would expect from Fig. 10 however the the $95 \%$ line degrades as increasingly indirect routes are added to the pool. The networks of NSFTEST instead seem to benefit from more route options until saturating at around $k=7$. Combined, these data show that if we were to choose a single value of $k$, our choice of 5 is a good compromise between both sets of networks.

\section{Comparison to ILP solution}

We shall now compare the lightpath establishment (LE) of sequential algorithms to that obtained by the ILP described in [6]. We note again that the ILP approach is a global optimization and hence represents the optimal solution to LE. The question is not whether we can guarantee to match it with a sequential alternative but rather how close can we get and what, if anything, do we sacrifice in the process. It should be also be noted that on larger networks the ILP approach quickly becomes infeasible - the BT22 topology in Fig. 6 took over 5 hours on a modern 8-core CPU - hence FF- $k$ SP is not truly competing with an ILP but with other sequential approaches 


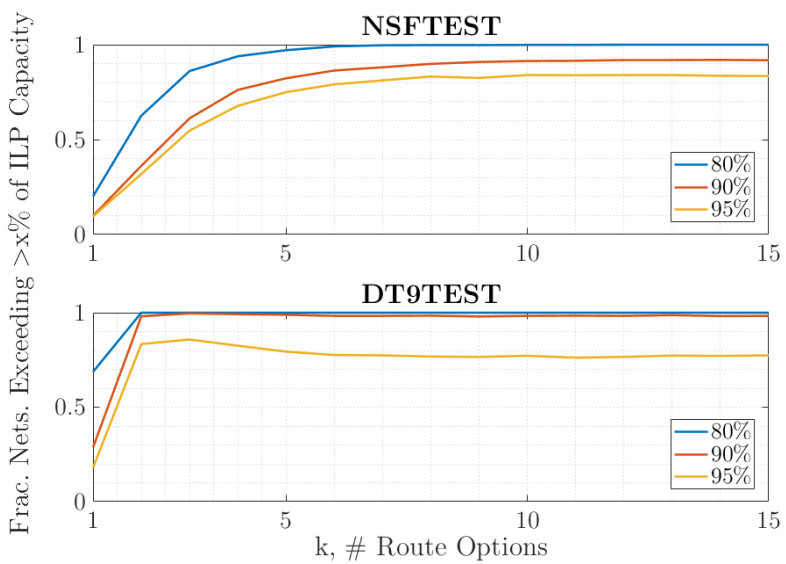

Fig. 15. FF- $k$ SP performance for various values of $k$ for NSFTEST, above, and DT9TEST, below.

that can scale to large networks such as those of the CoroNet project [34].

Given the cost function of the ILP, we should expect that when sequential algorithms achieve equivalent traffic levels they do so by employing more transceivers. The question is then how many more transceivers are required than optimal and how do other sequential approaches compare? When the sequential algorithms achieve capacity we can directly compare lightpath assignment with that of the ILP. By running $\mathrm{FF}-k \mathrm{SP}$ on the 1,000 networks of NSFTEST with up to 5 route options per demand we achieve capacity in 703 cases. On these networks we can count the number of transceivers required and compare it to the number required by the ILP solution; this is shown in Fig. 16. As expected, we see that the sequential algorithm typically requires more transceivers to achieve the same traffic levels however there is good agreement between the optimal value achieved with the ILP and that required by FF- $k \mathrm{SP}$, indeed the maximum difference is $\sim 12 \%$. This minor penalty is associated with using sequential LE when global optimization is an option.

On BT22, both FF- $k$ SP and CA-MU achieved capacity. We can therefore compare the solution from the ILP with the sequential algorithms when routing the exact same traffic. This comparison is shown in Table II, where the sequential algorithms had up to 5 route options per demand. We see that the ILP uses the fewest transceivers, which is expected given its cost function. But by doing so it uses more frequency slots than CA-MU which prefers to use more direct paths when uncongested. On both these metrics FF- $k \mathrm{SP}$ falls behind requiring both more frequency slots and more transceivers. One might then ask why ILP and CA-MU could not reach higher throughput? A possible explanation can be found in [11] which quantifies network fragmentation by assuming that contiguous spectrum is less fragmented than discontiguous. We see that although CA-MU might use fewer frequency slots the distribution of these slots is more fragmented and that although $\mathrm{FF}-k \mathrm{SP}$ uses the most frequency slots those it uses tend to be packed together more efficiently. This could explain why the two algorithms can load the same amount of traffic on a network despite their different approaches. Due to the

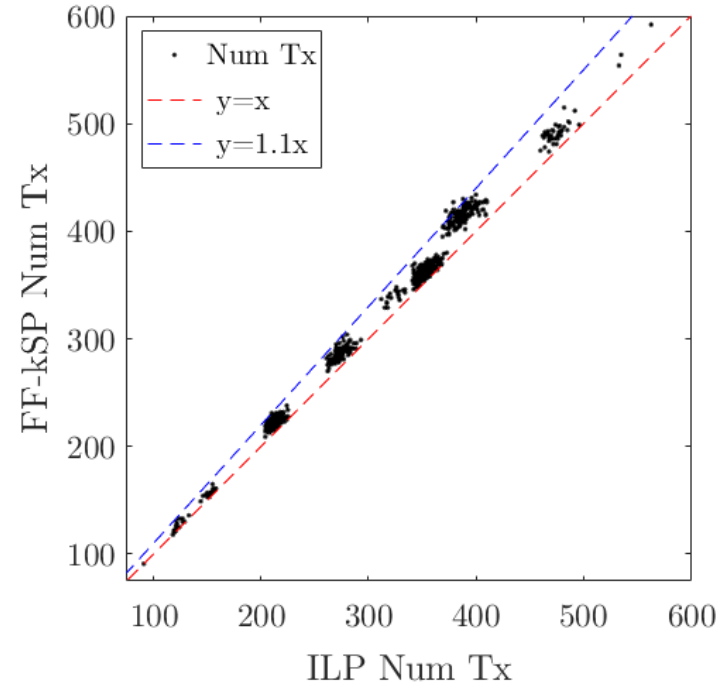

Fig. 16. Number of transceivers required by $\mathrm{FF}-k \mathrm{SP}$ relative to the ILP solution for 703 of 1,000 networks in NSFTEST that achieved capacity. The ILP minimizes this value however $\mathrm{FF}-k \mathrm{SP}$ shows good agreement and within $12 \%$ for all cases.

TABLE II

RWA COMPARISON FOR THE SAME TRAFFIC LEVEL ON BT22

\begin{tabular}{l|c|c|c}
\hline \hline & ILP & CA-MU & FF- $k$ SP \\
\hline Num Tx & 407 & 410 & 418 \\
Freq Slots Used & 1409 & 1314 & 1508 \\
Fragmentation [11] & 59.4 & 63.1 & 56.6 \\
\hline \hline
\end{tabular}

third part of the ILP cost function in Eq. (9), long distance and small SNR margin lightpaths are forced into the same few wavelengths. The assumption is that these wavelengths can then be labeled such that they are maximally separated to minimize NLI if we need to increase their transmission power. We do not perform this crucial step which explains why the ILP solution is less fragmented than that from CA-MU and we note that this is unlikely to remain true after relabeling.

\section{DISCUSSION}

This RWA algorithm represents an alternate way of approaching the problem and can be adapted to perform even better: this paper shows the simplest possible version to avoid unnecessary complications. By discriminating between path options (rather than simply providing the $k$ shortest) we can, no doubt, improve performance further. Exactly how we should achieve this requires further investigation; for example restricting certain demands to only one route option, as is often done by the ILP, might increase total network traffic at the expense of the resilience of network traffic to fiber cuts. We therefore require a balance between increasing traffic with real-world service provider considerations.

This algorithm can be of great use in fiber constrained scenarios by maximizing the traffic possible through a restricted section of spectrum. Such scenarios are common for example international expansion where a data center operator 
might need to lease limited spectrum from the incumbent national monopoly. Core networks are another case where fiber is limited due to the expense of laying often hundreds of kilometers between major cities.

The scalability and computational simplicity of this algorithm compared to that of an ILP-based approach, see Appendix, allows many network topologies to be tested in quick succession. Such a use case would enable improved network topologies by fast estimation of network capacity without the need for a global optimization approach which have much longer solve times per network, particularly in the worst case. These estimates might be especially useful to provide rankings in cases where various topologies are compared to each other and cardinal information is, while useful, not strictly necessary.

Our treatment of uniform traffic guarantees a near-uniform final traffic matrix which allows a direct comparison to ILP solutions. This cannot be achieved by selecting demands with uniform probabilities because the variance of the number of times each demand is selected increases linearly with the total traffic selected. We sample demands uniformly at random without replacement until each demand has been selected once, at which point we repeat the process; this continues until the network blocks. For NSFnet, uniform traffic between node pairs seems reasonable because the original network connected universities (or research institutes), however the DT9, DT14, and the BT22 networks are all reasonable representations of communication networks for the general public. This calls the uniform traffic assumption into question as the bandwidth required must surely be a function of the population associated with each node, as well as other geographic factors. Our algorithm, $\mathrm{FF}-k \mathrm{SP}$, would be unaffected by moving towards non-uniform traffic, however our traffic sampling approach would have to be adjusted. As with uniform probabilities, simply using non-uniform probabilities to sample demand requests is not enough to achieve the final traffic matrix we desire when the network blocks. Unfortunately, our 'selection without replacement' approach is unlikely to succeed, unaltered, because to achieve arbitrary ratios we would need to have duplicate demands in our sample: a ratio of 3 to 1 is straightforward, but 10 to 1 , or 100 to 1 , would soon resemble selecting with non-uniform probabilities because the network would block before all demands in the sample space were selected. Further work would be required to achieve a random, or pseudo-random, traffic sequence that results in arbitrary final traffic ratios; or failing that, one or many deterministic traffic sequences may be required.

\section{CONCLUSION}

We have introduced a wavelength and routing algorithm that focuses on wavelength packing over spectral efficiency. This algorithm, FF- $k \mathrm{SP}$, was shown to achieve increased traffic compared to previous algorithms by delaying blocking due to WDM conflicts. This more than makes up for the reduced spectral efficiency and we discuss how the inevitable latency penalties can be mitigated. We compared algorithm performance on a variety of real and simulated core network

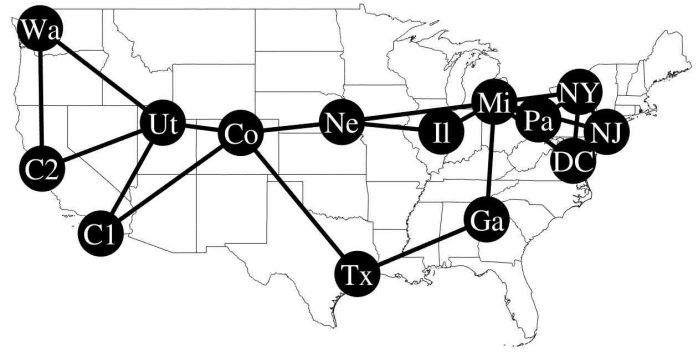

Fig. 17. NSFTEST ID 1

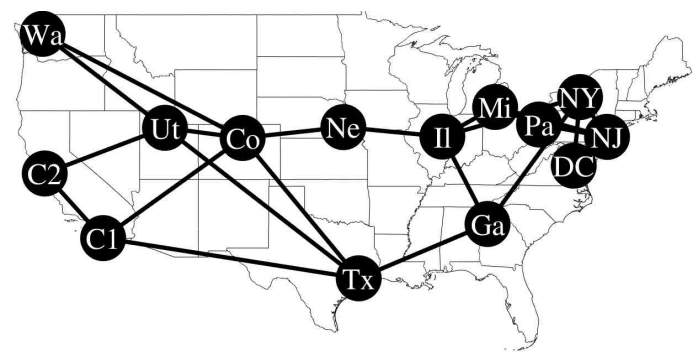

Fig. 18. NSFTEST ID 1000

topologies and confirm that the improvement is due to an algorithmic improvement and not an artifact from specific topologies. Due to an improved approach to uniform traffic we were able to compare sequential LE algorithms to ILP-based solutions. Once again, FF- $k$ SP outperforms other sequential algorithms and gave a good estimate of optimal capacity on most topologies while giving a scalable alternative to an ILP.

\section{APPENDIX \\ NETWORK TOPOLOGIES}

In this appendix we shall describe the properties of the network sets we generated based on the node locations of NSFnet, Fig. 3, and on DT9, Fig. 4. These were generated using a genetic algorithm to find topologies that minimized both fiber length and graph diameter (the longest shortest path). Additional requirements were that the network must be connected and that node degree was restricted to between 2 and 5 inclusive. The genetic algorithm recorded the 1,000 best topologies, given our cost function, in its archive and after running for some time the networks from this archive were used to populate the set of topologies used in this paper. This procedure was repeated for both NSFnet and DT9 resulting in 1,000 test topologies for each. Some example topologies from NSFTEST are given in Figs. 17 and 18. And examples from DT9TEST are given in Fig. 19.

The distribution of the number of links per network is given in Fig. 20 and we see, as expected, that the larger NSFnet topology requires more links to fulfill our requirements than DT9. We note that the number of links per network of NSFTEST is similar to that of the real-world topology on which they are based. This contrasts with DT9TEST, the networks in which have significantly fewer than the 17 links in the original DT9. This is because DT9 is highly meshed for a core network but it might indicate that on smaller networks, in densely populated areas, building costs are a smaller fraction 

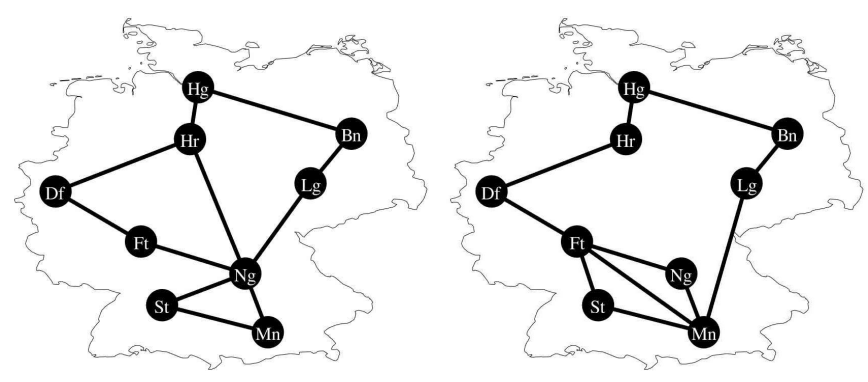

Fig. 19. DT9TEST ID 1, left, DT9TEST ID 1000, right.

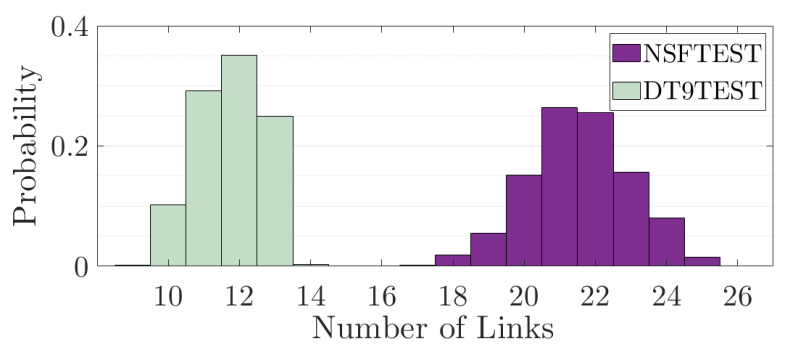

Fig. 20. Link distribution for the networks of NSFTEST, purple, and DT9TEST, light blue.

TABLE III

ILP SOLVE TIMES

\begin{tabular}{c|c|c|c|c}
\hline \hline & Total Time & Median & Worst Case & Prob $>6 \mathrm{hrs}$ \\
\hline DT9TEST & $17.5 \mathrm{hrs}$ & $19.3 \mathrm{~s}$ & $38.1 \mathrm{mins}$ & $4.2 \cdot 10^{-4}$ \\
NSFTEST & 5.7 days & $3.8 \mathrm{mins}$ & $3.1 \mathrm{hrs}$ & $7.3 \cdot 10^{-4}$ \\
\hline \hline
\end{tabular}

of total construction costs and in future we could allow for relatively more fiber deployment than on larger networks. For our purposes, of finding reasonable topologies on which to test algorithmic performance, the relatively few links on DT9TEST networks is of no concern.

We found the capacity of each network using the ILP approach described above. The time required to solve these ILPs are shown in Table III. We see that with a powerful system, running an Intel $17-6900 \mathrm{~K}$ at $3.8 \mathrm{GHz}$, the typical network solves quickly but the worst-case requires much more time. For NSFTEST the worst case took 50 times longer than the median and with DT9TEST it took over 100 times longer. The final column is extrapolated using extreme value statistics to estimate the likelihood of edge cases. In this case we see that the probability of an ILP requiring more than 6 hours to solve the RWA problem is non-zero for both network sets. When optimizing a topology we shall likely cycle through various prospective networks, far more than 1,000, and we are therefore highly likely to come across networks which take an impractically long time to solve. This makes it infeasible to use an ILP to estimate network capacity in this context.

\section{REFERENCES}

[1] J. M. Simmons, Optical Network Design and Planning. Springer, 2014.

[2] I. Chlamtac, A. Ganz, and G. Karmi, "Purely optical networks for terabit communication," in IEEE INFOCOM '89, Proc. 8th Ann. Conf. IEEE Comp. Commun. Soc., April 1989, pp. 887-896 vol.3.
[3] S. Boyd and L. Vandenberghe, Convex optimization. Cambridge University Press, 2004

[4] R. Ramaswami and K. N. Sivarajan, "Design of logical topologies for wavelength-routed all-optical networks," in Proceedings of INFOCOM'95, vol. 3, April 1995, pp. 1316-1325 vol.3.

[5] S. Subramaniam and R. A. Barry, "Wavelength assignment in fixed routing WDM networks," in Proc. ICC'97 - Int. Conf. Commun., vol. 1, June 1997, pp. 406-410 vol.1.

[6] D. J. Ives, P. Bayvel, and S. J. Savory, "Routing, modulation, spectrum and launch power assignment to maximize the traffic throughput of a nonlinear optical mesh network," Photonic Netw. Commun., vol. 29, no. 3, pp. 244-256, Jun 2015. [Online]. Available: https://doi.org/10.1007/s11107-015-0488-0

[7] A. Eira, J. Pedro, J. Pires, and J. P. Fernández-Palacios, "Performance evaluation of heuristic and ILP-based algorithms for multi-period planning of SBVT-enabled transport networks," in 2015 17th International Conference on Transparent Optical Networks (ICTON), Jul. 2015, pp. $1-6$.

[8] B. Mukherjee, D. Banerjee, S. Ramamurthy, and A. Mukherjee, "Some principles for designing a wide-area wdm optical network," IEEE/ACM Transactions on Networking, vol. 4, no. 5, pp. 684-696, Oct 1996.

[9] R. M. Rodriguez-Dagnino, E. O. Lopez-Caudana, H. Martinez-Alfaro, and J. L. Gonzalez-Velarde, "Simulated annealing and stochastic ruler algorithms for wavelength assignment planning in wdm optical networks," in IEEE SMC'99 Conference Proceedings. 1999 IEEE International Conference on Systems, Man, and Cybernetics (Cat. No.99CH37028), vol. 6, Oct 1999, pp. 1015-1020 vol.6.

[10] N. Banerjee, V. Mehta, and S. Pandey, "A genetic algorithm approach for solving the routing and wavelength assignment problem in wdm networks," in 3rd IEEE/IEE international conference on networking, ICN, 2004, pp. 70-78.

[11] P. Wright, M. C. Parker, and A. Lord, "Minimum- and maximum-entropy routing and spectrum assignment for flexgrid elastic optical networking [invited]," J. Opt. Commun. Netw., vol. 7, no. 1, pp. A66-A72, Jan 2015.

[12] A. Rubio-Largo et al., "A comparative study on multiobjective swarm intelligence for the routing and wavelength assignment problem," IEEE Transactions on Systems, Man, and Cybernetics, Part C (Applications and Reviews), vol. 42, no. 6, pp. 1644-1655, Nov 2012.

[13] H. Zang and J. P. Jue, "A review of routing and wavelength assignment approaches for wavelength-routed optical wdm networks," Opt. Netw. Mag., vol. 1, pp. 47-60, 2000.

[14] J. Y. Yen, "Finding the $\mathrm{k}$ shortest loopless paths in a network," Management Science, vol. 17, no. 11, pp. 712-716, 1971.

[15] S. J. Savory, "Congestion aware routing in nonlinear elastic optical networks," IEEE Photonic Tech. L., vol. 26, no. 10, pp. 1057-1060, 2014.

[16] R. Barry and S. Subramaniam, "The MAX SUM wavelength assignment algorithm for WDM ring networks," in Opt. Fiber Commun. Conf., Feb 1997, pp. 121-122.

[17] X. Zhang and C. Qiao, "Wavelength assignment for dynamic traffic in multi-fiber WDM networks," in Proc. 7th Int. Conf. Computer Commun. Netw., Oct 1998, pp. 479-485.

[18] R. J. Vincent, D. J. Ives, and S. J. Savory, "Estimating network throughput with an adaptive routing and wavelength assignment algorithm," in Opt. Fiber Commun. Conf. Optical Society of America, 2018, p. M2E.2. [Online]. Available: http://www.osapublishing.org/ abstract.cfm?URI=OFC-2018-M2E.2

[19] P. Poggiolini, "The GN model of non-linear propagation in uncompensated coherent optical systems," J. Lightwave Technol., vol. 30, no. 24, pp. 3857-3879, Dec 2012. [Online]. Available: http://jlt.osa.org/abstract.cfm?URI=jlt-30-24-3857

[20] G. Bosco et al., "Experimental investigation of nonlinear interference accumulation in uncompensated links," IEEE Photonic Tech. L., vol. 24, no. 14, pp. 1230-1232, July 2012.

[21] P. P. Mitra and J. B. Stark, "Nonlinear limits to the information capacity of optical fibre communications," Nature, vol. 411, pp. 1027 EP -, Jun 2001. [Online]. Available: http://dx.doi.org/10.1038/35082518

[22] D. Semrau, R. I. Killey, and P. Bayvel, "The gaussian noise model in the presence of inter-channel stimulated raman scattering," Journal of Lightwave Technology, vol. 36, no. 14, pp. 3046-3055, July 2018.

[23] D. J. Ives, "Coherent optical fibre networking in the nonlinear regime," Ph.D. dissertation, UCL, 12 2015. [Online]. Available: http://discovery.ucl.ac.uk/1473321/

[24] K. Cho and D. Yoon, "On the general BER expression of one- and twodimensional amplitude modulations," IEEE T. Commun., vol. 50, no. 7, pp. 1074-1080, July 2002. 
[25] D. J. Ives, P. Bayvel, and S. J. Savory, "Adapting transmitter power and modulation format to improve optical network performance utilizing the gaussian noise model of nonlinear impairments," Journal of Lightwave Technology, vol. 32, no. 21, pp. 4087-4096, Nov 2014.

[26] I. Roberts, J. M. Kahn, and D. Boertjes, "Convex channel power optimization in nonlinear wdm systems using gaussian noise model," Journal of Lightwave Technology, vol. 34, no. 13, pp. 3212-3222, July 2016.

[27] M. Scholten, T. Coe, and J. Dillard, "Continuously-interleaved bch (cibch) fec delivers best in class necg for $40 \mathrm{~g}$ and $100 \mathrm{~g}$ metro applications," in Opt. Fiber Commun. Conf., March 2010, pp. 1-3.

[28] G. Tzimpragos, C. Kachris, I. B. Djordjevic, M. Cvijetic, D. Soudris, and I. Tomkos, "A survey on fec codes for $100 \mathrm{~g}$ and beyond optical networks," IEEE Communications Surveys Tutorials, vol. 18, no. 1, pp 209-221, Firstquarter 2016.

[29] A. Betker et al., "Comprehensive topology and traffic model of a nationwide telecommunication network," J. Opt. Commun. Netw., vol. 6, no. 11, pp. 1038-1047, November 2014.

[30] S. D. Maesschalck et al., "Pan-European Optical Transport Networks: An Availability-based Comparison," Photonic Netw. Commun., vol. 5 , no. 3, pp. 203-225, May 2003. [Online]. Available: https://doi.org/10. 1023/A:1023088418684

[31] A. Mitra, D. Ives, A. Lord, S. Savory, S. Kar, and P. Wright, "Network equipment and their procurement strategy for high capacity elastic optical networks," J. Opt. Commun. Netw., vol. 8, no. 7, pp. A201A211, July 2016.

[32] P. Erdos and A. Rényi, "On the evolution of random graphs," Publ. Math. Inst. Hung. Acad. Sci, vol. 5, no. 1, pp. 17-60, 1960.

[33] B. Bollobás, Modern graph theory. Springer Science \& Business Media, 2013, vol. 184.

[34] A. L. Chiu et al., "Architectures and protocols for capacity efficient, highly dynamic and highly resilient core networks [invited]," J. Opt. Commun. Netw., vol. 4, no. 1, pp. 1-14, January 2012. 